\title{
Controle de qualidade aplicado a dados gravimétricos
}

\author{
Quality control applied to the gravity data
}

\author{
Gabriela Pasetto Falavigna ${ }^{1}$ \\ Sérgio Florêncio de Souza ${ }^{1}$ \\ Marilei Bender Xavier ${ }^{1}$ \\ Silvia Beatriz Alves Rolim ${ }^{1}$
}
${ }^{1}$ Departamento de Geodésia, Universidade Federal do Rio Grande do Sul - UFRGS. Av. Bento Gonçalves, 9500, Campus do Vale. Cep 91501-970. Porto Alegre, RS, Brasil. gabi_falavigna@hotmail.com, sergio.florencio@ufrgs.br, marileibx@hotmail.com, silvia.rolim@ufrgs.br

\begin{abstract}
RESUMO - O ato de medir é, essencialmente, um ato de comparar, que envolve erros derivados dos instrumentos, do operador, dos processos de medida, etc. Os erros presentes nos dados gravimétricos se propagam na determinação do modelo do geóide, por isso a sua identificação é de fundamental importância. Os objetivos desse trabalho consistiram na integração das informações gravimétricas coletadas por três Instituições e na detecção e remoção dos possíveis erros grosseiros desse banco de dados. Das observações que constituem o banco de dados gravimétricos do Estado do Rio Grande do Sul, 2.480 vieram do IAG/USP, 950 do IBGE e 6.071 observações do IG/UFRGS. O Estudo foi dividido em três etapas: verificação de dados duplicados (primeira etapa), onde foram identificadas 225 observações; análise da distribuição geográfica dos pontos (segunda etapa), onde foram encontradas 37 observações (0,60\% do banco de dados) com erro no posicionamento planimétrico; análise estatística (terceira etapa) baseada no desvio padrão dos dados. Os testes altimétricos e gravimétricos identificaram 284, 256 e 172 pontos suspeitos de conterem erros grosseiros, respectivamente, para as análises Global (4,58\% do banco de dados), Regional (4,13\% do banco de dados) e Local (2,77\% do banco de dados). Nos três tipos de análises realizadas, ou seja, Global, Regional e Local sete pontos foram identificados por dois testes.
\end{abstract}

Palavras-chave: erro grosseiro, análise estatística, gravidade, altimetria.

ABSTRACT - The act of measurement is, essentially, an act of comparing that involves errors derivatives of instruments, operator, processes of measurement, etc. The errors presents in the gravimetric data propagate on determination of the geoid model, so their identification is crucial. The objectives of this work consisted in integration of the gravimetric information collected by three Institutions and also in the detection and removal of possible blunders presents in this database. From observations that constitute the gravity database of Rio Grande do Sul, 2,480 came from IAG/USP, 950 from IBGE and 6,071 observations from IG/UFRGS. The Study was divided into three steps: check duplicate data (first step), which identified 225 observations; geographical distribution analysis of points (second step), where it was found 37 observations $(0.60 \%$ of database) with planimetric positioning error; statistical analysis (third step) based on the standard deviation of the data. The altimetric and gravimetric tests have identified 284,256 e 172 points suspects of present blunders, respectively, for the analysis (4.58\% of database), Regional (4.13\% of database) and Local (2.77\% of database). In the three types of analyzes, in other words, Global, Regional and Local seven points were identified by two tests.

Keywords: blunder, statistical analysis, gravity, altimetry.

\section{INTRODUÇÃO}

Os dados de gravidade são importantes nos estudos que visam o conhecimento da forma, da estrutura, das propriedades físicas e dos processos dinâmicos da Terra. Assim, a medida da gravidade, obtida na superfície da Terra, contém informações sobre o ponto onde a medição é realizada (aplicação geodésica), sobre a distribuição de massa no interior da Terra (aplicação geofísica) e, no caso de medições repetidas, sobre as variações temporais do corpo da Terra (aplicação geodinâmica).

A determinação da gravidade como qualquer outra medida geodésica pode conter erros. A detecção e remoção de erros grosseiros em bancos de dados gravimétricos são de fundamental importância, pois a existência de observações que contêm erros pode trazer problemas sérios no ajuste de modelos e na interpretação dos resultados. Quando se estuda determinado fenômeno, em que seja necessário utilizar um banco de dados, os eventuais erros grosseiros presentes nos dados podem mascarar resultados do estudo, fazendo com que o pesquisador tenha uma ideia errada à respeito do fenômeno em questão.

A detecção do erro grosseiro, a qual é o fato motivador deste trabalho, é essencial para que se obtenha uma precisão compatível com o método e o aparelho 
empregado. Os erros grosseiros, ao contrário dos erros sistemáticos e dos aleatórios, não são cumulativos nem apresentam uma distribuição de frequência normal quando o tamanho da amostra aumenta. Esse erro é um engano que pode ocorrer na medição ou nos cálculos (Vuolo, 1996) e pode ser causado por vários motivos, dentre eles, pode-se citar: leitura errada do operador, anotação errada dos dados medidos e erro de digitação dos dados de campo.

Este estudo foi dividido em três etapas: verificação da existência de dados duplicados (primeira etapa); análise da distribuição geográfica dos pontos para verificar a existência de possível erro no posicionamento planimétrico dos pontos (segunda etapa) e, por último, foi feita uma análise estatística (terceira etapa) baseada no desvio padrão dos dados. Na primeira etapa foram identificadas e eliminadas as observações duplicadas do banco de dados. Na segunda etapa, foram identificados os pontos que continham erro no posicionamento geográfico. Por fim, na terceira etapa, foi realizada uma comparação dos valores de aceleração da gravidade e de altitude ortométrica obtidos por duas técnicas diferentes, ou seja, avaliaram-se as diferenças entre os valores de altitude ortométrica obtidos a partir de um modelo topográfico e os valores de altitude ortométrica obtidos através dos meios convencionais de nivelamento e as diferenças entre os valores de aceleração da gravidade obtidos por modelos geopotenciais globais e os valores de aceleração da gravidade obtidos através de gravímetros. Para essa comparação, foram utilizados o modelo digital de elevação Shuttle Radar Topography Mission (SRTM) e o modelo geopotencial global Earth Gravitational Model 2008 (EGM2008). Nessa última etapa, foram feitas três tipos de análises: Global, Regional e Local.

\section{REVISÃO BIBLIOGRÁFICA}

\subsection{Método estatístico para detecção de erros grosseiros}

Um método utilizado para a detecção de erros grosseiros em banco de dados é a análise de resíduos, que consiste no estudo do comportamento do modelo no conjunto de dados observados, principalmente as discrepâncias entre os valores observados e os estimados. Na prática, para cada observação $i$ temos um erro aparente associado $\hat{e}_{i}$, que é a diferença entre o valor observado $z_{i}$ e o estimado $\hat{z}_{i}$, isto é (Gemael, 1994):

$$
\hat{\mathrm{e}}_{i}=z_{i}-\hat{z}_{i}
$$

Nesse trabalho, o erro aparente foi chamado de valor residual e consiste na diferença entre o valor da variável medida $\left(z_{i}\right)$, em um ponto no espaço, e o valor da interpolada $\left(\hat{Z}_{i}\right)$, no mesmo local, em uma superfície interpolada. O modelo de interpolação utilizado foi o Vizinho Natural (Natural Neighbor) e a interpolação dos dados foi feita no software Sufer.

\subsection{Suposição de uma distribuição normal dos resíduos}

A função gaussiana, também chamada função normal de erros, é muito utilizada para descrever erros experimentais. Verifica-se experimentalmente que, em geral, os erros seguem uma distribuição gaussiana com boa aproximação (Vuolo, 1996).

A importância da distribuição normal na teoria dos erros é explicada pelo teorema do limite central, que formula o seguinte:

As quantidades $y_{1}, y_{2}, \ldots, y_{N}$ são admitidas como $N$ variáveis aleatórias independentes que têm distribuição de probabilidades comum com valor médio verdadeiro finito $\mu$ e variância $\sigma^{2}$.

A quantidade $S_{N}^{*}$ é definida por

$$
S_{N}^{*}=\frac{S_{N}-N \mu}{\sigma \sqrt{N}}
$$

onde $S_{N}=y_{1}+y_{2}+\cdots+y_{N}$.

Uma vez que a variância correspondente à soma $S_{N}$ é $\sigma_{S}^{2}=\sigma^{2} N$, a quantidade $S_{N}^{*}$ pode ser interpretada como o número de desvios padrão $\sigma \sqrt{N}$ que a soma $S_{N}$ difere do valor médio populacional correspondente $(N \mu)$.

Indicando por $P\left(S_{N}^{*} \leq a\right)$ a probabilidade de que $S_{N}^{*}$ seja menor ou igual a um número finito qualquer $a$ $(-\infty \leq a \leq+\infty)$, o teorema do limite central estabelece que

$$
\lim _{N \rightarrow \infty} P\left(S_{N}^{*} \leq a\right)=\int_{-\infty}^{a} \frac{e^{-\frac{1}{2} z^{2}}}{\sqrt{2 \pi}} d z
$$

Em palavras, o teorema do limite central diz que a probabilidade de que $S_{N}^{*}$ seja menor ou igual a um valor qualquer $a$ é igual ao valor que teria esta probabilidade, se a distribuição de probabilidades para $S_{N}^{*}$ fosse uma distribuição normal padrão, que é uma distribuição gaussiana com valor médio $\mu=0$ e desvio padrão $\sigma=1$.

Considerando a definição de $S_{N}^{*}$, pode ser verificado que (Vuolo, 1996)

$$
P\left(S_{N} \leq a\right)=P\left(S_{N}^{*} \leq \frac{a-N \mu}{\sigma \sqrt{N}}\right) .
$$

A Eq. 3 no teorema do limite central também pode ser escrita na seguinte forma:

$$
\lim _{N \rightarrow \infty} P\left(\left|S_{N}^{*}\right| \leq a\right)=\Phi(a) \equiv \int_{-a}^{a} \frac{e^{-\frac{1}{2} z^{2}}}{\sqrt{2 \pi}} d z
$$

onde $a \geq 0$.

A Fig. 1 mostra o gráfico de $\Phi(a)$ em função de $a$. 


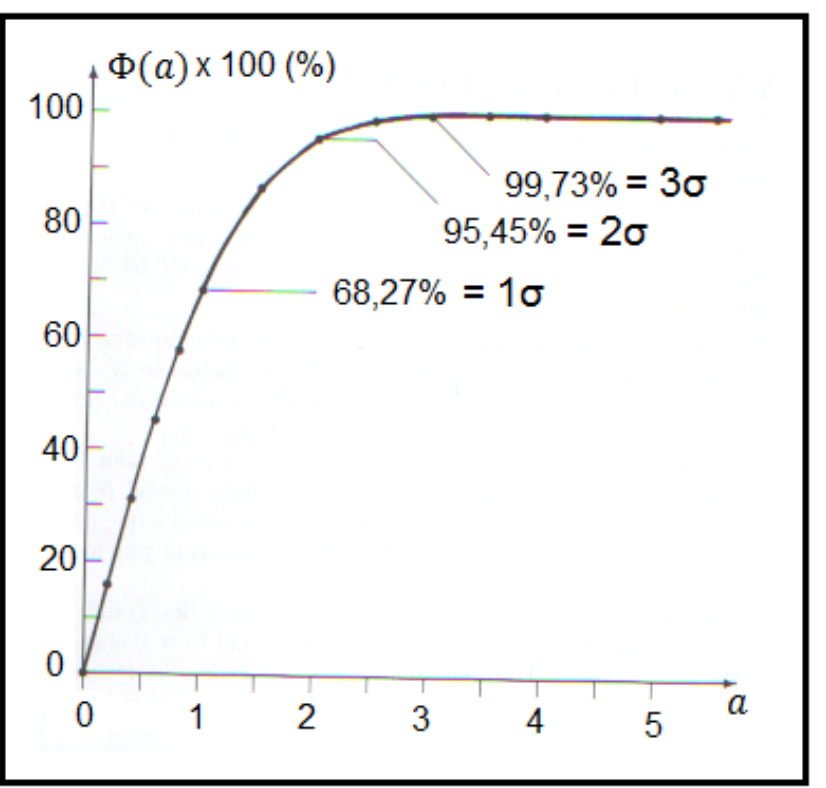

Figura 1 - Probabilidade $\Phi(a)$ x $a$

Fonte: Adaptado Vuolo (1996).

Um problema considerado frequente nas medições é o de estabelecer uma relação entre o "limite de erro" e a “incerteza padrão". Essa questão é considerada complicada e polêmica e não será detalhada nesse texto. Sendo assim, considerou-se um caso geral, a ser aplicado com bom senso, em cada análise particular (Vuolo, 1996).

Caso Geral: A distribuição de erros é considerada gaussiana (ou normal) e o limite de erro não é bem definido.

A incerteza em um resultado pode ser indicada de diferentes maneiras, entre as mais usuais, estão a incerteza padrão $(\sigma)$ e o limite de erro $(\mathrm{L})$. A incerteza padrão pode ser definida como o desvio padrão da distribuição de erros e sua interpretação é baseada no conceito de intervalo de confiança. Define-se intervalo de confiança como sendo o intervalo estimado de um parâmetro estatístico; quanto maior a probabilidade de o intervalo conter o parâmetro, maior será o intervalo de confiança; é usado para indicar a confiabilidade de uma estimativa e a probabilidade de essas estimativas estarem corretas é determinada pelo coeficiente de confiança $P$.

O limite de erro (L) é definido como o valor máximo que pode ter o erro $\eta$. No caso de uma distribuição de erros simétrica, que se anula além de certo valor $\mathrm{y}=\mathrm{L}$, o limite de erro é dado por:

$$
-\mathrm{L}<\mathrm{\eta}<+\mathrm{L} \text { com confiança } \mathrm{P}=100 \% \text {. }
$$

No caso de uma curva normal, que é simétrica em relação à origem e a área total sob a curva é considerada igual a 1 ou $100 \%$, não existe um limite de erro absoluto, pois a gaussiana nunca se anula teoricamente. Entretanto, a Fig. 3 mostra que a gaussiana tende a zero para erros maiores que $\mathrm{L}=3 \sigma$. Quando se trata de erros estatísticos, essa quantidade é denominada limite de erro estatístico e é definido por
$\mathrm{L}_{\mathrm{e}}=3 \sigma_{m}$

onde $\sigma_{m}$ é o desvio padrão no valor médio $\bar{y}$.

Se os erros estatísticos seguem uma distribuição normal, as probabilidades para a variável aleatória normal podem ser dadas por áreas sob a curva (Fig. 2). As porcentagens de valores em alguns intervalos são:

- $68,26 \%$ dos valores de uma variável aleatória normal estão contidos no intervalo compreendido entre as distâncias de um desvio padrão acima e um desvio padrão abaixo da média.

- $95,45 \%$ dos valores de uma variável aleatória normal estão contidos no intervalo compreendido entre as distâncias de dois desvios padrão acima e dois desvios padrão abaixo da média.

- $\quad 99,73 \%$ dos valores de uma variável aleatória normal estão contidos no intervalo compreendido entre as distâncias de três desvios padrão acima e três desvios padrão abaixo da média.

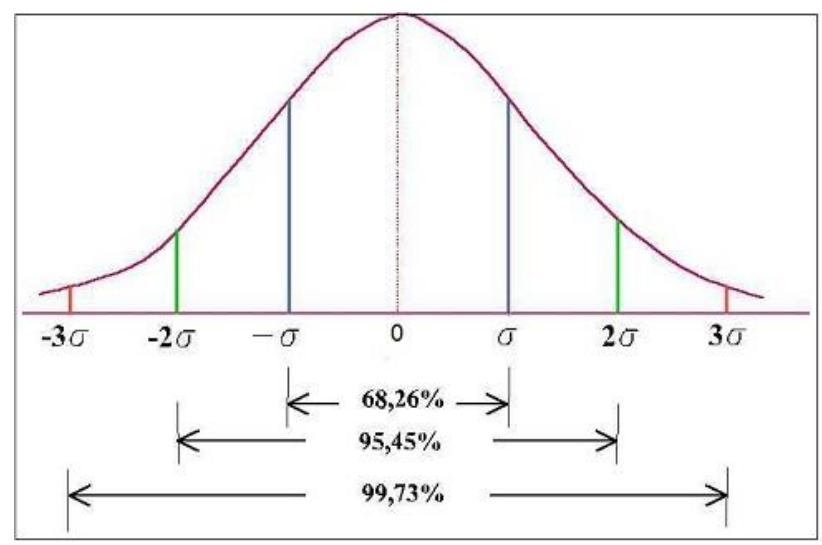

Figura 2 - Curva da distribuição gaussiana (ou Normal).

Fonte: http://pt.wikipedia.org/wiki/Intervalo_de_confian\%C3\%A7a.

Nesse trabalho, foi considerado que a distribuição dos resíduos (altimétricos e gravimétricos) dos pontos segue a distribuição normal. Sendo assim, foi possível utilizar a Eq. 7 para calcular os limiares de corte dos testes estatísticos realizados com esses dados e, assim, estabelecer um intervalo com um alto coeficiente de confiança $(99,73 \%)$, importante para a interpretação dos resultados alcançados.

\section{METODOLOGIA}

\section{1. Área de estudo}

A área de estudo (Fig. 3), para a realização desse trabalho, situa-se na porção sul do Brasil e corresponde ao Estado do Rio Grande do Sul (RS). O RS abrange uma área total de 281.730,223 $\mathrm{km}^{2}$ (IBGE Estados, 2013) e 
está compreendido entre as latitudes $33^{\circ} 43^{\prime} \mathrm{S}$ e $27^{\circ} 05^{\prime} \mathrm{S}$ e as longitudes $49^{\circ} 42^{\prime} \mathrm{W}$ e $57^{\circ} 40^{\prime} \mathrm{W}$.

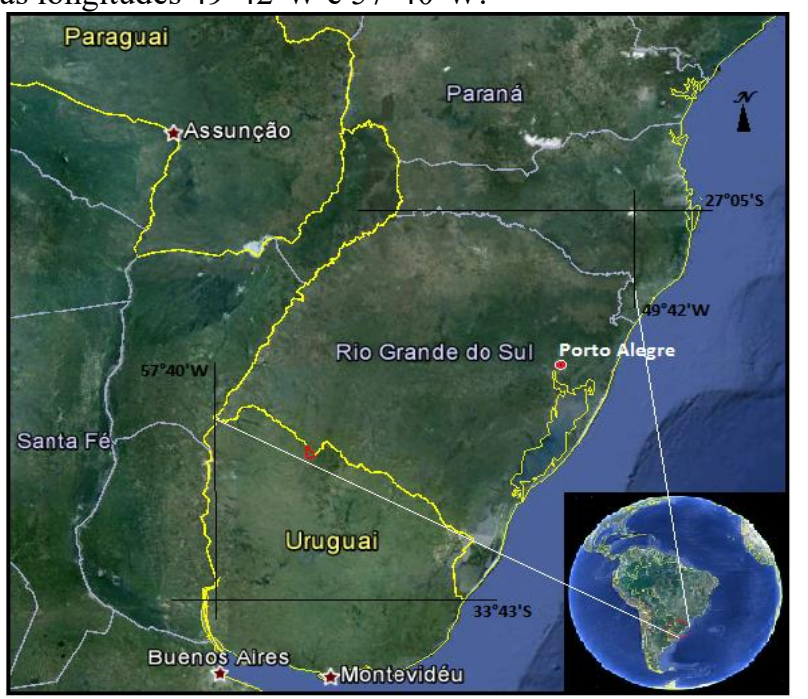

Figura 3 - Mapa da localização da Área de Estudo.

Fonte: Adaptado de Xavier (2009).

\subsection{Dados utilizados}

Os valores de aceleração da gravidade dos pontos levantados pelo Instituto de Geociências da Universidade Federal do Rio Grande do Sul (IG/UFRGS), pelo Instituto de Astronomia, Geofísica e Ciências Atmosféricas da Universidade de São Paulo (IAG/USP) e pelo Instituto Brasileiro de Geografia e Estatística (IBGE) foram obtidos através de gravímetros; as altitudes ortométricas através dos métodos clássicos de nivelamento e a posição geográfica de alguns pontos foi determinada através da interpolação de coordenadas de cartas topográficas nas escalas 1:50.000, 1:100.000 e 1:250.000 (IBGE, 2012).

\subsubsection{Dados gravimétricos terrestres}

A malha gravimétrica do RS é resultado de inúmeros levantamentos geofísicos executados por Universidades (IG/UFRGS, UFPR, IAG/USP), órgãos e empresas públicas (IBGE, Petrobrás, CPRM, DNPM, ON/CNPq). Uma parte dos dados levantados por essas instituições integra o acervo de dados geofísicos do Banco Nacional de Dados Gravimétricos Terrestres (BNDG). As estações gravimétricas contém um conjunto de informações sobre o ponto levantado, entre elas, a posição geográfica, a altitude ortométrica e a aceleração da gravidade.

O Estado do Rio Grande do Sul apresenta uma boa cobertura gravimétrica nas regiões noroeste e centro-sul do estado e uma cobertura insuficiente na região nordeste. As observações levantadas pelo IG/UFRGS, pelo IAG/USP e pelo IBGE correspondem respectivamente a, 6071, 2480 e 950 pontos. No entanto, é importante ressaltar que os pontos levantados pelo IAG/USP (efetivamente utilizados nesse trabalho) correspondem somente às observações levantadas no RS; dos 2480 pontos levantados por essa Instituição, 1793 foram levantados no RS e fazem parte do banco de dados. A localização espacial dos pontos levantados pelas três
Instituições, utilizados neste estudo, pode ser verificada na Fig. 4.

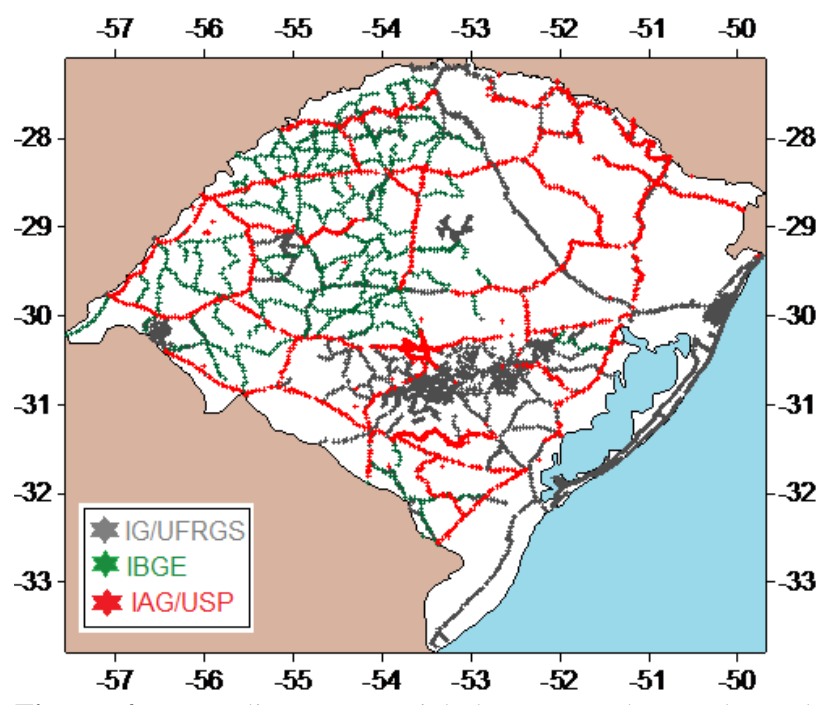

Figura 4 - Localização espacial dos pontos levantados pelo IG/UFRGS, IAG/USP e IBGE.

\subsubsection{Dados gravimétricos do EGM2008}

Os Modelos Geopotenciais Globais (GGM), resultados de conjuntos de coeficientes do potencial anômalo desenvolvido em série de funções harmônicas esféricas, são determinados pela integração de dados obtidos por uma grande variedade de instrumentos e técnicas observacionais. Atualmente, os modelos digitais do geopotencial integram os sistemas de referência geodésicos e são usados, rotineiramente, como esferóides para a representação do campo de gravidade anômalo de alta resolução (Souza et al., 2008).

O modelo geopotencial Earth Gravitational Model 2008 (EGM2008) foi divulgado na Assembleia Geral da União Europeia de Geociências (European Geosciences Union - General Assembly 2008), ocorrida em Viena, na Áustria, em 2008 (Nunes, 2010). O EGM2008 contém coeficientes do potencial gravitacional expresso em harmônicos esféricos até o grau e ordem 2159, resultando numa resolução espacial aproximada de 5' de arco; contém também coeficientes adicionais que podem estender o seu grau até 2190 e ordem 2159 (Pavlis et al., 2012); esses coeficientes são compatíveis com o sistema geodésico WGS84 e estão na forma de modelos gravimétricos digitais. Saatkamp et al. (2009), na sua avaliação do modelo geiodal EGM2008, estimou que o erro médio absoluto da ondulação geoidal, para o Brasil, gerado por esse GGM, foi de $35 \mathrm{~cm}$ aproximadamente.

Nesta pesquisa, o cálculo do geoide com os dados do EGM2008 foi feito através do programa disponível no sítio do International Centre for Global Earth Models (http://icgem.gfz-

potsdam.de/ICGEM/potato/Service.html). Para esse cálculo, utilizou-se até o grau e ordem 2159, o que garante a resolução espacial aproximada de 5' de arco $(\sim 9,5 \mathrm{~km})$ sobre a superfície terrestre. Não foi utilizado um grau maior devido a não existirem estudos ainda que 
avaliem a qualidade dos dados gerados quando calculados com um grau maior.

\subsubsection{Dados altimétricos terrestres}

A obtenção das informações altimétricas utilizadas nesse trabalho se deram através dos métodos clássicos de nivelamento: nivelamento geométrico, trigonométrico e barométrico. A precisão desses métodos varia de $0,1 \mathrm{~m}$ a $10 \mathrm{~m}$ dependendo do método empregado e do equipamento utilizado (IBGE, 2012).

As informações planimétricas de alguns pontos do banco de dados foi determinada por meio da interpolação de coordenadas de cartas topográficas nas escalas 1:50.000, 1:100.000 e 1:250.000, o que implica numa acurácia da posição geográfica dos pontos de algumas centenas de metros, dependendo da escala da carta $(0,5$ $\mathrm{km}, 1 \mathrm{~km}$ e $2,5 \mathrm{~km}$, respectivamente) (IBGE, 2012). Em áreas de topografia acidentada, em que a elevação varia muito em poucos metros, qualquer erro na planimetria resultará discrepante da informação altimétrica obtida em campo e a derivada do modelo digital de elevação adotado; o que implica na deterioração da precisão da informação altimétrica.

\subsubsection{Dados altimétricos do SRTM}

O SRTM (Shuttle Radar Topographic Mission) é um projeto internacional liderado pela National GeospatialIntelligence Agency (NGA) e pela National Aeronautics and Space Administration (NASA) com o objetivo de produzir um modelo topográfico digital de alta resolução para a Terra (NASA, 2013). Essa missão foi executada pelo ônibus espacial Endeavour, durante o período de 11 a 22 de fevereiro de 2000, cobrindo cerca de $80 \%$ da superfície terrestre (área compreendida entre as latitudes $60^{\circ} \mathrm{N}$ e $56^{\circ} \mathrm{S}$ ), com resolução espacial de um segundo de arco (1"), o que corresponde a aproximadamente 30 metros. O método de aquisição dos dados altimétricos usado foi a interferometria por radar, que consiste na comparação de duas imagens de radar tomadas de pontos ligeiramente diferentes para obtenção da elevação. Para a realização desse procedimento, o ônibus espacial Endeavour foi equipado com um mastro de 60 metros e duas antenas de recepção (banda $\mathrm{C}$ e banda $\mathrm{X}$ ) que foram instaladas uma no compartimento de carga do ônibus espacial e a outra na ponta do mastro que se estendia para fora da espaçonave; isso possibilitou a aquisição dos dados em uma mesma órbita, garantindo assim a melhor qualidade dos mesmos (Rodriguez et al., 2005). As imagens do SRTM possuem resolução de 30 metros para os Estados Unidos e 90 metros para os outros países; seu referencial altimétrico é o elipsóide WGS84 e seu modelo terrestre é representado pelo Earth Gravitational Model 1996 (EGM96).

Uma avaliação da qualidade dos dados altimétricos do SRTM feita para o Estado do RS pode ser encontrada em Lemos et al. (2004) e para o Brasil e a Argentina em Blitzkow et al. (2007). Os resultados preliminares da análise da qualidade dos dados altimétricos derivados do
SRTM para uma área dentro do Estado do RS, obtidos por Lemos et al. (2004), demonstraram que, para muitas aplicações, o SRTM pode substituir os MDTs obtidos a partir de mapas topográficos na escala 1:250.000.

\subsection{Validação do banco de dados gravimétricos}

O procedimento de validação do banco de dados constituiu-se de 4 etapas: montagem do banco de dados e eliminação de dados repetidos; análise visual gráfica para detecção de possíveis erros grosseiros, a partir da visualização da distribuição espacial dos pontos; geração de resíduos utilizando os dados do SRTM (teste 1) e do EGM2008 (teste 2); análise estatística e interpretação dos resultados.

A elaboração do banco de dados gravimétricos do RS a partir da integração dos pontos amostrados das três Instituições resultou num total de 9.501 pontos. A tabela de observações contém as seguintes informações dos pontos: latitude, longitude, altitude ortométrica e aceleração da gravidade. Após a união dos dados, foi realizada uma análise para eliminação de observações repetidas, a partir da verificação da latitude e longitude dos pontos, na qual foram removidos 225 pontos. $\mathrm{Na}$ segunda etapa, foi feita uma análise visual gráfica para a remoção de pontos que apresentavam erro em seu posicionamento geográfico (planimetria), que resultou na exclusão de 37 pontos do banco de dados; a localização espacial desses pontos pode ser verificada na Fig. 5. Realizadas as duas primeiras etapas, partiu-se para a detecção de possíveis erros grosseiros através da geração de resíduos altimétricos e gravimétricos.

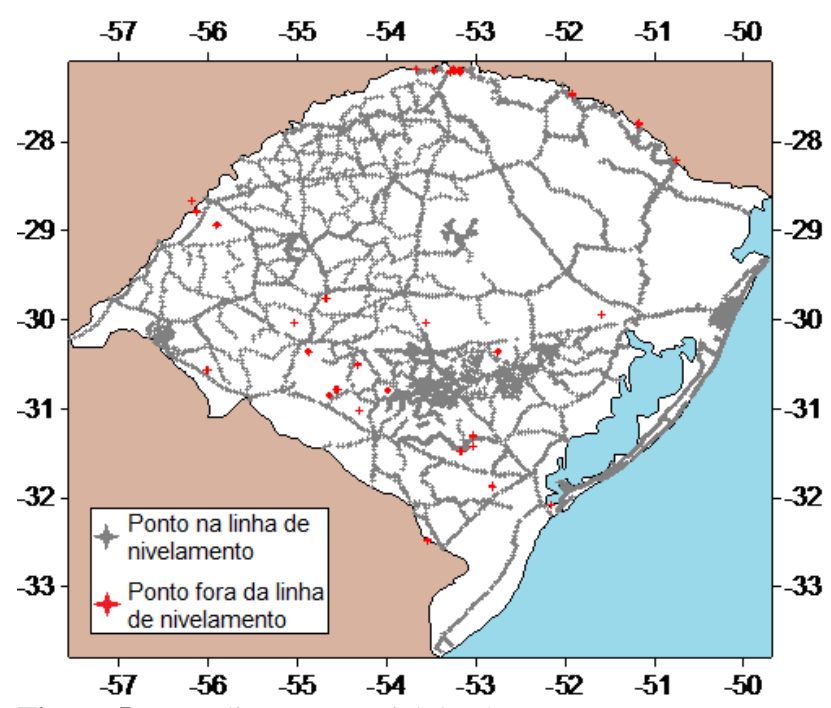

Figura 5 - Localização espacial dos 37 pontos com erro no posicionamento geográfico (planimetria).

\subsubsection{Definição dos limiares de corte}

A definição dos limiares de corte foi feita com base no item 2.4 e na interpretação dos resíduos altimétricos e gravimétricos gerados. Os resíduos altimétricos consistem na comparação das altitudes ortométricas do SRTM com as altitudes ortométricas obtidas em campo através dos 
métodos convencionais de nivelamento (teste 1) e os resíduos gravimétricos consistem na comparação do modelo geoidal obtido com os dados do EGM2008 com as acelerações da gravidade obtidas por gravímetros (teste 2).

\subsubsection{Análises realizadas}

Foram realizadas três tipos de análises: Global, Regional e Local.

A análise Global consistiu na avaliação de toda a área do Rio Grande do Sul, sem compartimentá-la. A Regional consistiu na avaliação da área do RS dividida em 4 regiões e uma dessas regiões (a que continha o maior número de observações) dividida em mais outras 3 sub-regiões (Fig. 6) e a análise Local consistiu no compartimento da extensão do RS em áreas de aproximadamente $1^{\circ} \times 1^{\circ}$, o que resultou em 35 áreas.

Para a análise Global, a partir dos resíduos gerados, foram produzidos histogramas residuais da altitude e da gravidade para uma melhor visualização da distribuição de frequência desses resíduos; foram produzidos também mapas para a localização espacial dos pontos identificados em cada teste.

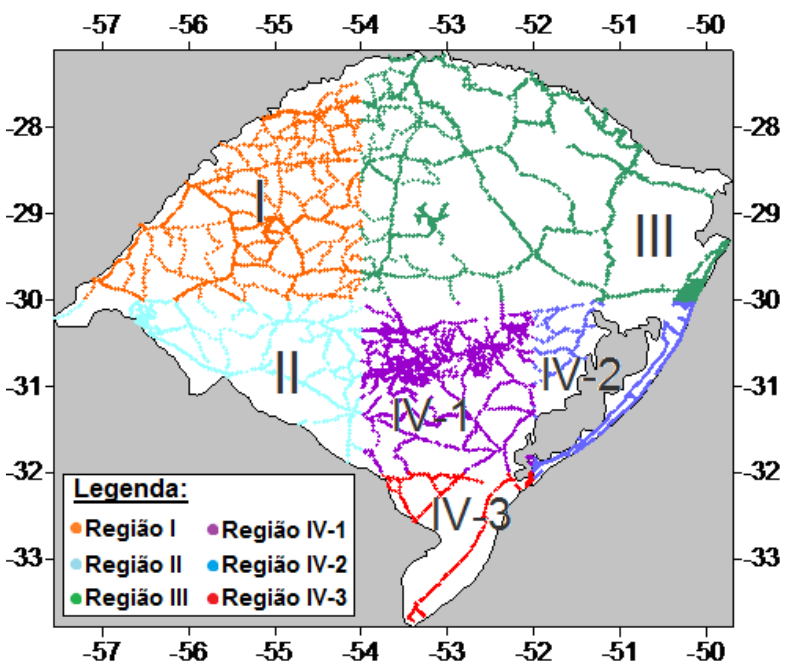

Figura 6 - Divisão e subdivisão da área do RS.

\section{RESULTADOS E DISCUSSÃO}

\subsubsection{Análise Global}

Os resultados estatísticos alcançados com a aplicação dos testes no banco de dados podem ser verificados na Tab. 1.

Tabela 1 - Estatísticas referentes às 6.164 observações dos testes residuais

\begin{tabular}{cccccc}
\hline $\begin{array}{c}\text { Valores } \\
\text { residuais }\end{array}$ & $\begin{array}{c}\mathbf{N}^{\mathbf{0}} \\
\text { pontos }\end{array}$ & Mínimo & Máximo & Média & $\begin{array}{c}\text { Desvio } \\
\text { Padrão }\end{array}$ \\
\hline $\begin{array}{c}\text { Altimétricos } \\
\text { (teste 1) } \\
\text { metro }\end{array}$ & 6164 & $-250,99$ & 793,47 & $-2,63$ & 23,21 \\
$\begin{array}{c}\text { Gravimétricos } \\
\text { (teste 2) } \\
\text { mGal }\end{array}$ & 6164 & $-369,39$ & 112,61 & $-2,60$ & 12,66 \\
\hline
\end{tabular}

Através dos valores máximos e mínimos percebe-se que há uma distribuição irregular tanto dos resíduos altimétricos como dos gravimétricos e os altos desvios padrão apresentados indicam que há dispersão dos dados em relação à média.

Utilizando a Eq. 7 e os desvios padrão mostrados na Tab. 1, foram calculados os limiares de corte, os quais podem ser observados na Tab. 2.

Tabela 2 - Limiares de corte adotados devido aos dois testes realizados

\begin{tabular}{ccc}
\hline Resíduos & $\begin{array}{c}\text { Mínimo } \\
\text { (limiar de } \\
\text { corte) }\end{array}$ & $\begin{array}{c}\text { Máximo } \\
\text { (limiar de } \\
\text { corte) }\end{array}$ \\
\hline $\begin{array}{c}\text { Altimétricos (teste 1) } \\
\text { metro }\end{array}$ & -70 & +70 \\
$\begin{array}{c}\text { Gravimétricos (teste 2) } \\
\text { mGal }\end{array}$ & -38 & +38 \\
\hline
\end{tabular}

Considerando o gráfico representado na Fig. 7, notase a falta de normalidade (seguimento de uma distribuição normal) dos resíduos altimétricos e a presença de valores extremos localizados à esquerda da distribuição. Os pontos que apresentavam valores residuais altimétricos abaixo de $-70 \mathrm{~m}$ e acima de $+70 \mathrm{~m}$ foram considerados possíveis erros grosseiros. Com base nisso, foram identificados 226 pontos (3,64\% do banco de dados) suspeitos de conter erros grosseiros.

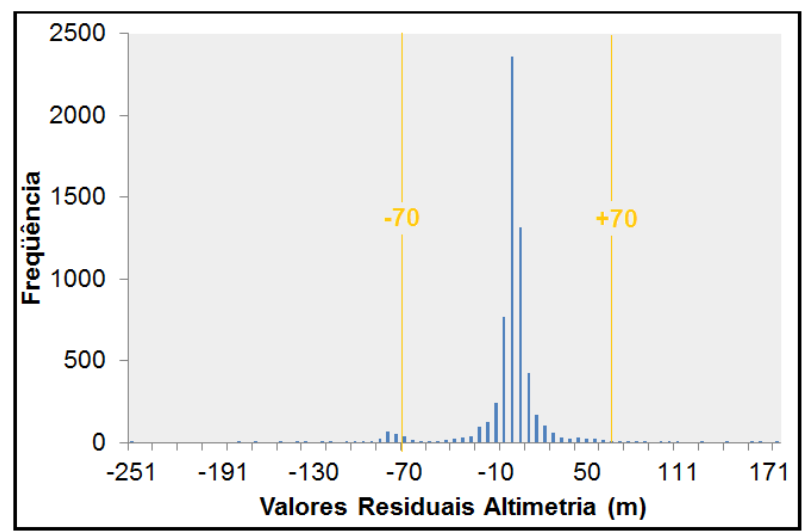

Figura 7 - Histograma dos valores residuais altimétricos entre as 6.164 observações e os respectivos pontos do SRTM.

Com base no histograma representado na Fig. 8, pode-se perceber que a distribuição dos resíduos gravimétricos também não segue uma distribuição normal, pois se verifica a presença de uma tendência à direita da distribuição. No entanto, a distribuição dos valores residuais gravimétricos se aproxima bem mais de um comportamento normal do que a distribuição dos valores residuais altimétricos (Fig. 7). Os pontos que apresentavam valores residuais gravimétricos abaixo de $38 \mathrm{mGal}$ e acima de $+38 \mathrm{mGal}$ foram considerados possíveis erros grosseiros. Sendo assim, foram identificados 65 pontos (1,04\% do banco de dados) suspeitos de conter erros grosseiros. 


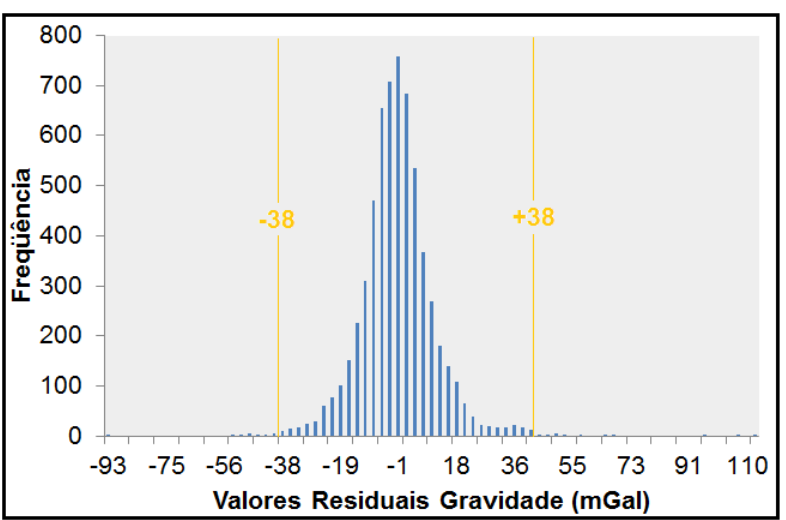

Figura 8 - Histograma dos valores residuais gravimétricos entre as 6.164 observações e os respectivos pontos do EGM2008.

Após essas interpretações, reuniram-se em uma planilha as 284 observações suspeitas de conter erros grosseiros, identificadas pela aplicação dos testes 1 e 2 no banco de dados (a visualização da distribuição espacial das 284 observações pode ser verificada na Fig. 9). Com isso, verificou-se que 7 pontos $(0,11 \%$ do banco de dados $)$ foram identificados nos dois testes.

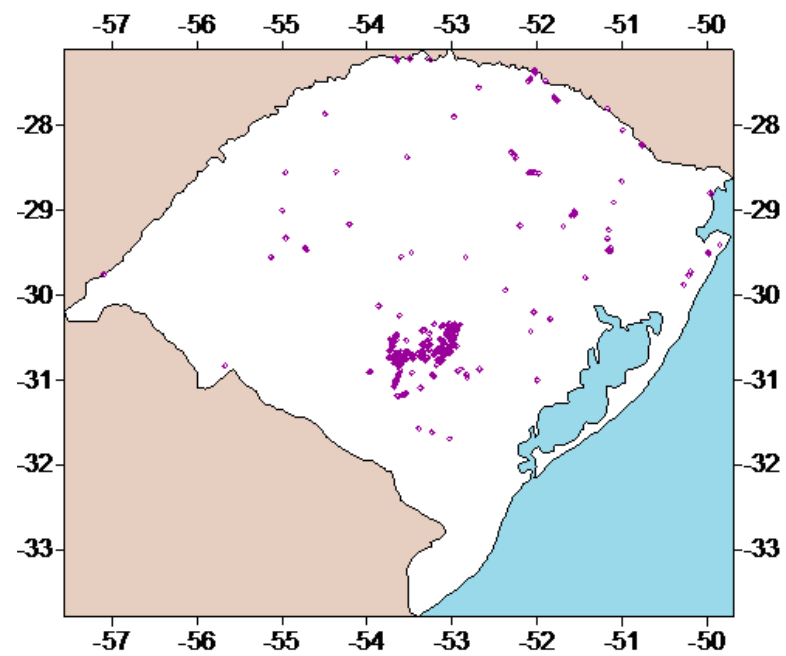

Figura 9 - Localização espacial dos 284 pontos suspeitos de conter erros grosseiros identificados pelos limiares de corte altimétricos e gravimétricos.

Na Tab. 3 é possível verificar o comportamento da distribuição dos dados após a eliminação dos pontos considerados erros grosseiros.

Tabela 3 - Estatísticas referentes após a eliminação dos 284 pontos

\begin{tabular}{cccccc}
\hline $\begin{array}{c}\text { Valores } \\
\text { residuais }\end{array}$ & $\begin{array}{c}\mathbf{N}^{\mathbf{0}} \\
\text { pontos }\end{array}$ & Mínimo & Máximo & Média & $\begin{array}{c}\text { Desvio } \\
\text { Padrão }\end{array}$ \\
\hline $\begin{array}{c}\text { Altimétricos } \\
\text { (teste 1) } \\
\text { metro }\end{array}$ & 5880 & $-69,92$ & 69,47 & $-0,13$ & 12,38 \\
$\begin{array}{c}\text { Gravimétricos } \\
\text { (teste 2) } \\
\text { mGal }\end{array}$ & 5880 & $-37,52$ & 37,61 & $-2,59$ & 10,50 \\
\hline
\end{tabular}

Analisando a Tab. 3, a Fig. 10 e a Fig. 11, nota-se uma melhora nos parâmetros dos valores residuais altimétricos e gravimétricos em relação aos obtidos anteriormente (vide Tab. 1); ambas as distribuições residuais se aproximaram mais da curva normal após a eliminação dos pontos considerados erros grosseiros; em termos de porcentagem, as distribuições residuais ficaram por volta de $5 \%$ mais próximas da curva normal. Sendo assim, há uma melhora geral nos parâmetros dos dois testes após a eliminação dessas observações.

Os limiares altimétricos e gravimétricos adotados nessa análise (70 m e $38 \mathrm{mGal}$, respectivamente) foram superiores ao erro esperado na precisão absoluta de modelos digitais de elevação do $\operatorname{SRTM}(17 \mathrm{~m})$ e de observações altimétricas coletadas em campo $(0,1 \mathrm{~m}$ a 10 m) e ao pior erro que se pode cometer em observações gravimétricas (15 mGal a $20 \mathrm{mGal}$ ) (Xavier, 2009).

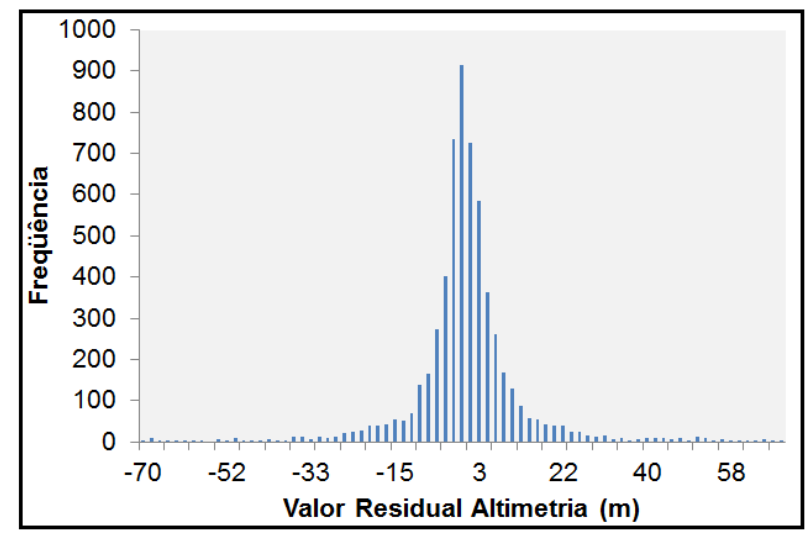

Figura 10 - Histograma dos valores residuais altimétricos entre as 5.880 observações e os respectivos pontos do SRTM.

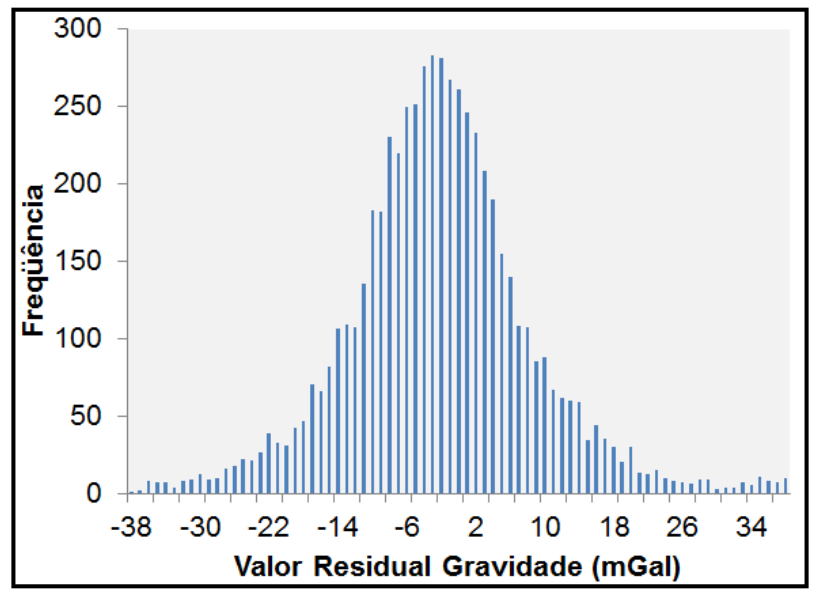

Figura 11 - Histograma dos valores residuais gravimétricos entre as 5.880 observações e os respectivos pontos do EGM2008.

\subsubsection{Análise Regional}

Devido ao número de pontos identificados na análise Global, verificou-se a possibilidade de avaliar a detecção de erros grosseiros por regiões; com a finalidade de verificar se a aplicação dos testes estatísticos em áreas menores influencia na quantidade de pontos identificados como erros grosseiros. O número de pontos detectados, nessa análise, com a aplicação dos dois testes pode ser verificado no Quadro 1. 


\begin{tabular}{|c|c|c|}
\hline Região & Testes & $\begin{array}{c}\text { Pontos } \\
\text { identificados }\end{array}$ \\
\hline \multirow{4}{*}{ I } & Teste 1 & 22 \\
\hline & Teste 2 & 14 \\
\hline & Testes 1 e 2 & 3 \\
\hline & Total & 39 \\
\hline \multirow{4}{*}{ II } & Teste 1 & 12 \\
\hline & Teste 2 & 10 \\
\hline & Testes 1 e 2 & 0 \\
\hline & Total & 22 \\
\hline \multirow{4}{*}{ III } & Teste 1 & 20 \\
\hline & Teste 2 & 12 \\
\hline & Testes 1 e 2 & 2 \\
\hline & Total & 34 \\
\hline \multirow{4}{*}{ IV } & Teste 1 & 135 \\
\hline & Teste 2 & 24 \\
\hline & Testes 1 e 2 & 2 \\
\hline & Total & 161 \\
\hline \multirow{4}{*}{ IV-1 } & Teste 1 & 18 \\
\hline & Teste 2 & 9 \\
\hline & Testes 1 e 2 & 1 \\
\hline & Total & 28 \\
\hline \multirow{4}{*}{ IV-2 } & Teste 1 & 7 \\
\hline & Teste 2 & 4 \\
\hline & Testes 1 e 2 & 1 \\
\hline & Total & 12 \\
\hline \multirow{4}{*}{ IV-3 } & Teste 1 & 15 \\
\hline & Teste 2 & 7 \\
\hline & Testes 1 e 2 & 3 \\
\hline & Total & 25 \\
\hline
\end{tabular}

Quadro 1 - Número de pontos identificados por Região devido à aplicação dos testes 1 e 2

Conforme o Quadro 1 e considerando as quatro regiões (I, II, III, IV) foram identificados 256 pontos suspeitos de conter erros grosseiros, ou seja, 28 pontos a menos que na análise Global. Desses pontos, 205 coincidem com os identificados na análise Global e dos 7 pontos identificados na análise anterior por ambos os testes, 4 coincidem com os detectados depois do compartilhamento da área. Na Região IV foram identificados 161 pontos suspeitos de conterem erros grosseiros, onde 2 pontos foram identificados por ambos os testes. Com a subdivisão dessa área em 3 subáreas, foram detectados 65 pontos suspeitos, onde 5 pontos foram identificados por ambos os testes. Desses 65 pontos, 29 coincidem com os identificados antes da subdivisão e dos 5 pontos detectados pelos dois testes 1 coincide com os identificados anteriormente.

Da mesma forma, analisando a Região IV foram detectados 161 pontos suspeitos e depois da subdivisão dessa região em três sub-regiões foram identificados 65 pontos suspeitos (96 pontos a menos).

\subsubsection{Análise Local}

Nessa análise foram identificados 172 pontos suspeitos (112 pontos a menos dos identificados pela análise Global e 84 pontos a menos dos identificados pela análise Regional). Desses 172 pontos suspeitos, 78 coincidem com os identificados na análise Global e dos 7 pontos detectados pelos dois testes 4 pontos coincidem com os identificados nas análises Regional e Global. Dos 205 pontos identificados coincidentes nas análises Regional e Global, 61 coincidem com os identificados na análise Local.

Sendo assim, concluiu-se que somente os 65 pontos identificados comuns às três análises (Global, Regional e Local) podem ser considerados erros grosseiros e devem ser removidos do banco de dados. Com isso, obteve-se um banco de dados gravimétricos com 6.099 pontos. A distribuição espacial dos 65 e dos 6.099 pontos pode ser verificada, respectivamente, na Fig. 12 e na Fig. 13.

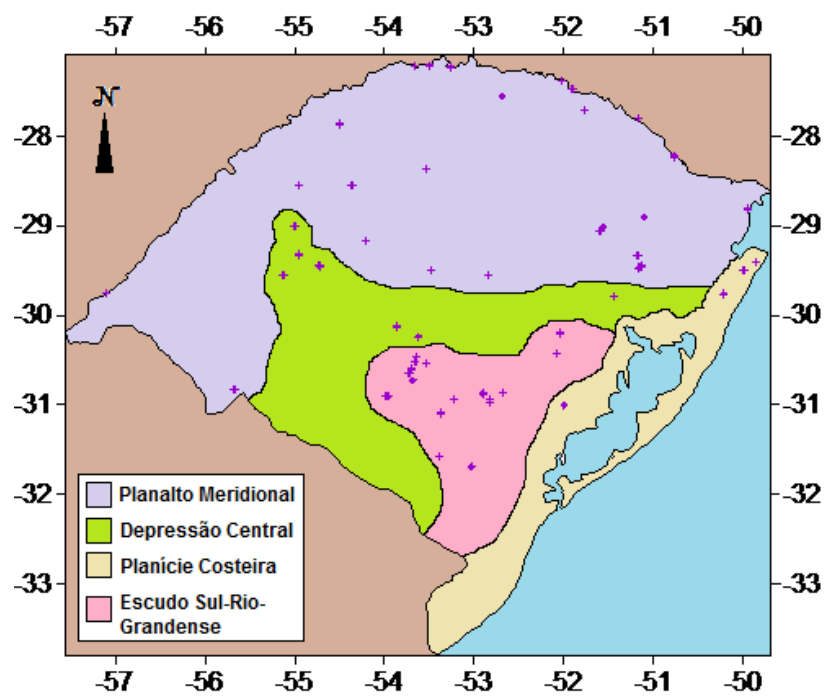

Figura 12 - Localização espacial dos 65 pontos identificados comuns às análises Global, Regional e Local.

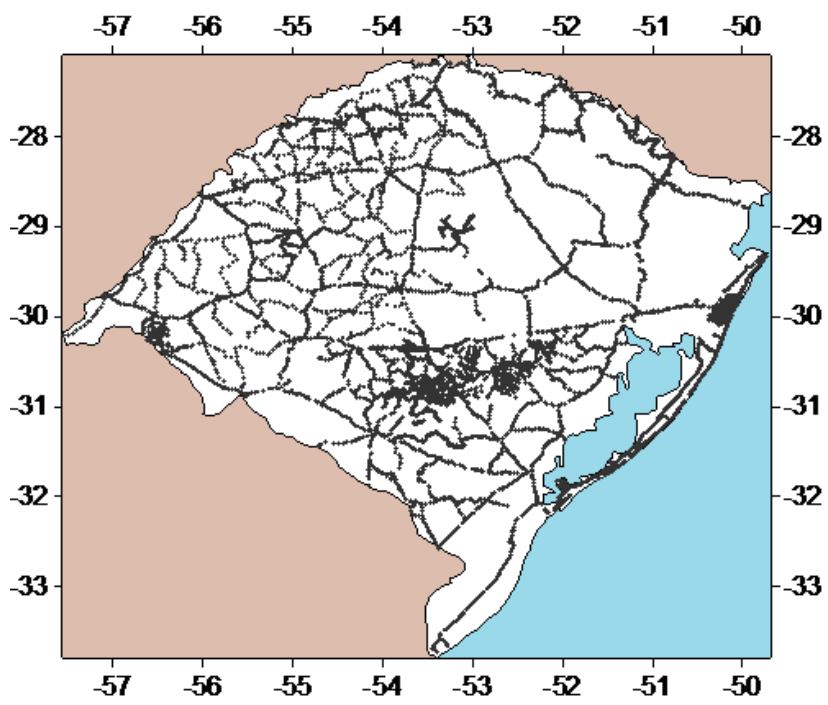

Figura 13 - Distribuição espacial dos 6.099 pontos do banco de dados gravimétricos do Rio Grande do Sul.

Com base na Fig. 12, verifica-se que os pontos identificados localizados na região de maior altitude 
(região nordeste do RS) provavelmente apresentam erros relacionados à altimetria terrestre, devido à falta de Referências de Nível (RRNN) nessa região ou à incorreta aquisição das altitudes. Já os pontos localizados em altitudes mais baixas (região da Planície Costeira, Depressão Central e oeste do Planalto Meridional) possivelmente apresentam erros associados à planimetria, devido à extração de coordenadas interpoladas de cartas topográficas, o que implica numa precisão de algumas centenas de metros (dependendo da escala da carta) e à geologia da região, pois as características geológicas interferem nos valores da aceleração da gravidade. Notase também que é na região do Escudo Sul-Rio-Grandense onde está localizada a maior concentração dos pontos prováveis de conter erros grosseiros. Acredita-se que a localização desses pontos se deva à geologia muito complexa encontrada nessa região: relevo ondulado com grande variação altimétrica e vertentes com afloramento rochoso e cristas; além disso, a região do Escudo é constituída por rochas ígneas do período Pré-Cambriano, apresentando rochas metamórficas na região do Complexo Canguçu e rochas magmáticas na região das Suítes Graníticas e apresenta também vertentes íngremes com manto de alteração pouco espesso (IFCRS/UFSM, 2013). Essas características geológicas encontradas nessa região, provavelmente contribuem para a ocorrência de erros nas medições planimétricas, altimétricas e gravimétricas dos pontos.

\section{CONCLUSÕES}

O âmago desse trabalho é a determinação de um modelo geoidal com a melhor resolução possível. Para isso, uma avaliação profunda à respeito dos erros dos dados gravimétricos deve ser feita, levando em consideração os erros devidos aos data verticais, aos dados GPS sobre o nivelamento, à variação de densidade topográfica, aos GGMs e aos MDTs. Se essa avaliação não for feita, nunca será possível chegar a um geóide com resolução centimétrica.

Assim, o método da análise de resíduos para detecção de possíveis erros grosseiros no banco de dados gravimétricos do Estado do Rio Grande do Sul mostrouse uma importante ferramenta, auxiliando na validação das observações medidas pelas três Instituições (IAG/USP, IBGE e IG/UFRGS). Os dois testes realizados baseados nos resíduos altimétricos e gravimétricos mostraram-se eficientes na localização dos erros grosseiros. A definição dos limiares de corte, através da Eq. 7, para cada teste tornou possível a identificação dos pontos suspeitos de conter esses erros. A subjetividade na eliminação desses pontos foi necessária, pois a distribuição inicial dos dados impediu a obtenção de um modelo normal para ser possível a eliminação de erros grosseiros.

Concluiu-se que os resultados obtidos são coerentes, pois a maior probabilidade de ocorrência de erros na altimetria é em áreas de topografia acidentada; as posições geográficas extraídas de cartas topográficas podem originar erros na planimetria dos pontos, desviando-os das linhas de nivelamento e as discrepâncias identificadas nos valores da aceleração da gravidade podem ter relação com a geologia da área em questão, pois a aceleração gravitacional sofre influência direta da distribuição irregular das densidades das rochas em subsuperfície.

Concluiu-se também que a aplicação do critério adotado para a definição dos limiares de corte $(3 \sigma)$ depende da extensão da área em estudo, pois quanto menor for a área avaliada menor será o número de pontos identificados como suspeitos. Assim, deve existir uma extensão de área mínima a ser considerada para que a aplicação desse método não se torne ineficaz.

\section{AGRADECIMENTOS}

Este trabalho faz parte de um projeto mais amplo sobre a determinação e avaliação do geóide no Estado do Rio Grande do Sul. Esse projeto conta com o apoio do CNPQ na forma de fomento a projeto de pesquisa, através do apoio financeiro recebido em forma de Bolsa de Iniciação Científica PIBIC/CNPq-UFRGS.

\section{REFERÊNCIAS BIBLIOGRÁFICAS}

BLITZKOW, D.; MATOS, A. C. O. C. \& CINTRA, J. P. 2007. SRTM evaluation in Brazil and Argentina with emphasis on the Amazon region. International Association of Geodesy Symposia. v.130, p. 266-271. Disponível <http://link.springer.com/chapter/10.1007\%2F978-3540-49350-1 40\#>. Acesso em 26/11/2013.

IBGE. 2012. Sistema Geodésico Brasileiro. Disponível em:

<http://www.ibge.gov.br/home/geociencias/geodesia/g ravimetrica.shtm $>$. Acesso em 20/12/2012.

IBGE Estados. 2013. Rio Grande do Sul. Disponível em: $<$ http://www.ibge.gov.br/estadosat/perfil.php?sigla=rs $>$. Acesso em 08/11/2013.

IFCRS/UFSM. 2013. Relevo. Disponível em: <http://coralx.ufsm.br/ifcrs/relevo.htm>. Acesso em 08/01/2013.

GEMAEL, C. Introdução ao ajustamento de observações: aplicações geodésicas. Curitiba: Editora da UFPR, 1994. 319p.

LEMOS, M. C.; SOUZA, S. F. \& ROCHA, R. S. 2004. Avaliação da qualidade dos dados altimétricos derivado do Shuttle Radar Topographic Mission (SRTM): Resultados Preliminares. Anais I Simpósio em Ciências Geodésicas e Tecnologias da Geoinformação, Recife, 01-03 setembro 2004. Disponível em: <http://www.ufpe.br/cgtg/ISIMGEO/CD/html/Fotogra metria\%20e\%20Sensoriamento\%20Remoto/Artigos/f 008.pdf >. Acesso em 26/11/2013.

NASA. 2013. Shuttle Radar Topography Mission. Disponível em: <http://www2.jpl.nasa.gov/srtm/>. Acesso em 24/01/2013.

NUNES, V. C. 2010. Testes sobre a eficiência do Modelo do Geopotencial EGM2008 na cidade de Porto Alegre. 
Porto Alegre. 96p. Dissertação de Mestrado em Sensoriamento Remoto, Programa de Pós-graduação em Sensoriamento Remoto, Centro de Estudos e Pesquisa em Sensoriamento Remoto e Meteorologia, Universidade Federal do Rio Grande do Sul.

PAVLIS, N. K.; HOLMES, S. A.; KENYON, S. C. \& FACTOR, J. K. 2012. The development of the Earth Gravitational Model 2008 (EGM2008). Journal of Geophysical Research, vol. 117, B04406.

RODRIGUEZ, E.; MORRIS, C. S.; BELZ, J. E.; CHAPIN, E. C.; MARTIN, J. M.; DAFFER, W. \& HENSLEY, S. 2005. An assessment of the SRTM topographic products. Technical Report JPL D-31639, Jet Propulsion Laboratory. Pasadena, California. $143 \mathrm{p}$.

SAATKAMP, E. D.; MORAES, C. V. \& FARRET, J. C. 2009. Avaliação do modelo geoidal EGM2008 para o Brasil. Anais XIV Simpósio Brasileiro de Sensoriamento Remoto, Natal, 25-30 abril 2009, p. 4303-4310. Disponível em: <http://marte.sid.inpe.br/col/dpi.inpe.br/sbsr\%4080/20 08/11.17.20.21/doc/4303-4310.pdf $>$. Acesso em 25/11/2013.

SOUZA, S. F.; MATSUOKA, M. T.; GOTZ, J. J. \& SEVERO, T. C. 2008. Sobre o Novo Modelo do Geopotencial EGM2008. In: Sérgio Florêncio de Souza; Marcelo Tomio Matsuoka. (Org.). Série em Geomática: 10 anos do Curso de Engenharia Cartográfica da UFRGS. 1ed. Porto Alegre: Instituto de Geociências, UFRGS, v.2, p. 67-73.

VUOLO, J.H. 1996. Fundamentos da Teoria de Erros. Editora Edgard Blücher Ltda. São Paulo, 249p.

XAVIER, M.B. 2009. Detecção de erros grosseiros em banco de dados gravimétrico terrestre do Estado do Rio Grande do Sul. Porto Alegre. 103p. Dissertação de Mestrado em Sensoriamento Remoto, Centro Estadual de Pesquisa em Sensoriamento Remoto e Meteorologia, Universidade Federal do Rio Grande do Sul. 